\title{
Modelling the Discrete-Cell Gravure Roll Coating Process
}

\author{
A New Perspective \\ R. W. Hewson ${ }^{\text {a }}$, N. Kapur, and P. H. Gaskell \\ School of Mechanical Engineering, University of Leeds, Leeds. LS2 9JT. UK.
}

\begin{abstract}
New results for the process of discrete-cell, reverse mode gravure coating are presented, with the model considering a single cell and taking account of web thickness (stiffness) - an important feature neglected in previous analyses. Key results are: (i) confirmation that web tension, over the range encountered industrially, has negligible influence on web deformation; (ii) that web tension plays a secondary role to that of web stiffness. For parameters characteristic of those encountered in a typical coating operation the level of web deformation is found to be small, alluding to the importance of the two different length scales in the overall process - that at the cell (small) and coating bead (large) level.
\end{abstract}

\section{Introduction}

Discrete-cell gravure roll coating involves the transfer of fluid, via a thin coating bead [1], from indentations in a roll surface (typically laser engraved or knurled) to a substrate, or web (typically plastic but including metal) in close proximity, as the two sweep past each other. The reverse mode of operation, considered here, is the one in most common use due to its superior stability characteristics [2].

Early modelling work in the area includes the simple model of Rees [3] for the case of a single cell moving beneath a pinned free surface; a later model by Schwartz [4] considered the case of multiple cells. A related two dimensional analysis by Powell et al. [5] allowed imposition of the roll-to-web gap, web velocity and cross-cell pressure gradient so as to explore their influence on the emptying of rectangular cells. Although these approaches fail to adequately replicate gravure coating as they do not model the entire process (but rather the upstream meniscus based on a given roll to web gap and flow rate), they do reveal that: (i) an increase in cell size results in greater fluid pickout (the ratio of volume of fluid leaving the coating bead to that entering via the cells); (ii) the alignment of a gravure cell at an angle relative to the mean flow increases pickout.

Latterly, Yin \& Kumar [6] have derived a model, for the two-dimensional flow between a flexible web and a patterned surface containing a single cell, based on lubrication theory with the web treated as either a tensioned or a compliant surface, or a combination of the two. In treating a tensioned web their analysis assumes it to have zero thickness and as such stiffness is neglected. When the relevant gravure cell length scale is considered then web thickness is not insignificant. They subsequently refined their model [7], using Stokes' equations rather than lubrication theory for the flow in the coating bead. Nevertheless, their earlier assumptions about a negligible web thickness remains and in the second paper they assume highly sub-ambient pressures within the fluid (four orders of magnitude less than atmospheric) and unrealistically

a e-mail: r.w.hewson@leeds.ac.uk 




(a)

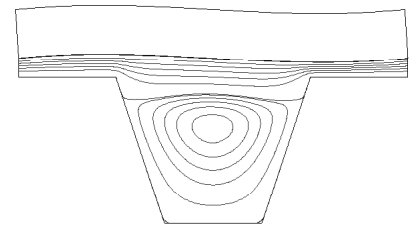

(b)

Fig. 1: (a) Problem domain considered in this analysis, (b) shows typical result for a pickout of 0.6, $E=2.4 \times 10^{7}$ (web deformation is multiplied by 300 for illustrative purposes)

high web tensions; the deformation of the web is therefore dominated by the mean sub-ambient pressures with the fluid flow making negligible difference to the web deformation.

This paper investigates the flow within a single cell (as shown in figure 1) and examines the deformation of the web over the length of the cell, for a given pickout and roll-to-web gap. It is therefore of fundamental importance in developing an understanding of the length scales over which web deformation occurs.

\section{Problem Specification and Method of Solution}

Analysis is confined to Newtonian liquids and a solid domain associated with a single gravure cell located well within the entirety of the coating bead. Stokes flow is assumed (due to the low Reynolds number involved), governed by:

$$
0=\nabla \cdot\left[-p \mathbf{I}+\left(\nabla \mathbf{u}+(\nabla \mathbf{u})^{T}\right)\right] ; \quad \nabla \cdot \mathbf{u}=0
$$

where, $\mathbf{u}=\mathbf{U} / U_{\text {roll }}$ and $p=P A / \eta U_{\text {roll }}$, with $\mathbf{U}$ the fluid velocity, $U_{\text {roll }}$ the gravure roll speed, $\eta$ the fluid viscosity ( $\mathbf{I}$ is the identity matrix) and $P$ the fluid pressure. The cell opening, $A$, is used throughout the analysis to non-dimensionalise the problem - this, and other dimensions, are shown in figure 1(a). The non-dimensional web velocity, given by $S+1$ where $S$ is the speed ratio defined as $S=U_{\text {web }} / U_{\text {roll }}$, is imposed in a direction parallel of the web surface, thereby accounting for the web deformation. The upstream and downstream boundary conditions are prescribed such that the velocities are periodic while the downstream pressure is uniformly shifted from that of the upstream pressure boundary distribution, thereby giving periodicity in the pressure. This shift in the pressures is a function of required pickout, $\Phi$, calculated in terms of the flow rate, $q$, between the web and roll, namely: $\Phi=q /$ (average cell depth) (and by considering the change in the frame of reference). Web deformation is modelled through the application of Green strains, $\varepsilon_{i j}$, rather than simpler strain-displacement relationships, which account for web tension effects:

$$
\begin{aligned}
\varepsilon_{x} & =\frac{1}{2}\left(\frac{\partial \zeta_{x}}{\partial x}+\frac{\partial \zeta_{x}}{\partial x}+\frac{\partial \zeta_{x}}{\partial x} \cdot \frac{\partial \zeta_{x}}{\partial x}+\frac{\partial \zeta_{y}}{\partial x} \cdot \frac{\partial \zeta_{y}}{\partial x}\right) \\
\varepsilon_{x y}=\varepsilon_{y x} & =\frac{1}{2}\left(\frac{\partial \zeta_{x}}{\partial y}+\frac{\partial \zeta_{y}}{\partial x}+\frac{\partial \zeta_{x}}{\partial x} \cdot \frac{\partial \zeta_{x}}{\partial y}+\frac{\partial \zeta_{y}}{\partial x} \cdot \frac{\partial \zeta_{y}}{\partial y}\right) \\
\varepsilon_{y} & =\frac{1}{2}\left(\frac{\partial \zeta_{y}}{\partial y}+\frac{\partial \zeta_{y}}{\partial y}+\frac{\partial \zeta_{x}}{\partial y} \cdot \frac{\partial \zeta_{x}}{\partial y}+\frac{\partial \zeta_{y}}{\partial y} \cdot \frac{\partial \zeta_{y}}{\partial y}\right)
\end{aligned}
$$






Fig. 2: Maximum displacement of the web for a range of pickouts and roll-to-web gaps, $E=2.4 \times 10^{7}$, for web tension of $T=500,000$. Contour values $\times 10^{-6}$

where $\zeta_{x}$ and $\zeta_{y}$ are the displacements in $x$ and $y$ respectively. Assuming the web to be a linearly elastic isotropic material, enables the stress-strain relationship to be written thus:

$$
\sigma=D \varepsilon+\sigma_{0}, \quad \text { where } \quad D=\frac{E}{(1+\nu)(1-2 \nu)}\left[\begin{array}{ccc}
1-\nu & \nu & 0 \\
\nu & 1-\nu & 0 \\
0 & 0 & \frac{1-2 \nu}{2}
\end{array}\right], \sigma=\left[\begin{array}{c}
\sigma_{x} \\
\sigma_{y} \\
\sigma_{x y}
\end{array}\right], \varepsilon=\left[\begin{array}{c}
\varepsilon_{x} \\
\varepsilon_{y} \\
\varepsilon_{x y}
\end{array}\right] \text {. }
$$

where $\sigma$ is the stress, $D$ is the elasticity matrix and $\sigma_{0}$ is the initial stress within the material. $E$ and $\nu$ are the non-dimensional Young's modulus (related to the dimensional Young's modulus $E^{\prime}$ by $\left.E=\frac{E^{\prime} A}{\eta U_{\mathrm{rol}}}\right)$ and Poisson's ratio respectively. The principal of virtual work is then applied to close the problem. The boundary constraints enforced are: where fluid and web are in contact the web experiences viscous and pressure forces; an unconstrained boundary condition is applied along the upper air-side of the web; repeated boundaries for displacement are imposed at the upstream and downstream ends.

The fluid equations (1) were solved within a moving mesh framework (coordinates $x_{m}, y_{m}$ ) with respect to a reference frame $(x, y)$ using an Arbitrary Lagrangian Eulerian (ALE) formulation, the obvious choice for the application concerned. At all boundaries of the fluid domain, with the exception of the lower fluid-side of the web, zero mesh displacement was imposed; displacement of the lower fluid-side of the web was governed by the web displacement, with Laplace smoothing used to ensure a regularly space mesh. Solution of the coupled problem was achieved via the Comsol Multiphysics finite element package (www.comsol.com). A rectangular mesh having 2000 elements and 29748 degrees of freedom was used, with mesh independence confirmed by systematic comparison of the results obtained with corresponding ones generated using a mesh containing 8000 elements with 113084 degrees of freedom.

\section{Results}

Overall, the effect of a wide range of parameters has been examined. These include the rollto-web gap, fluid viscosity, web tension and Young's modulus. A typical result showing the associated flow pattern and web deformation is given in figure 1(b). The major result, however, enables the range of the web deformation on a cellular length scale to be assessed and its importance to the overall coating process to be judged. Figure 2 shows web deformation as a function of $g\left(=\frac{G}{A}\right)$ and $\Phi$. For the range of tensions examined the web displacement remains insensitive to the web tension. The plots reveal that as the gap, $g$, decreases then web deformation increases - as expected due to the increased pressures within the gap required to obtain the specified pickouts. Note that the maximum pressure difference encountered across the cell is of the order of $10^{4}$, which, for this particular cell size, leads to a pressure gradient of the same order. In practice, if such a large pressure gradient were present within the coating bead it would be far in excess of what could be supported by the curvature of the web wrapped around the gravure roll. The line of minimum pressure difference across the cell for each roll-to-web 




(a)

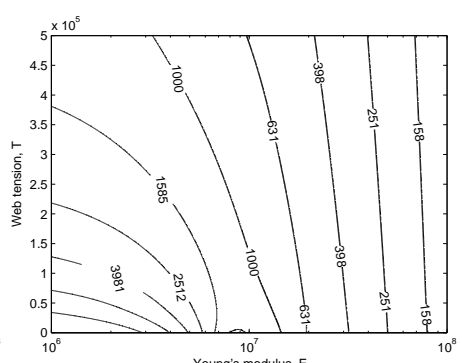

(b)

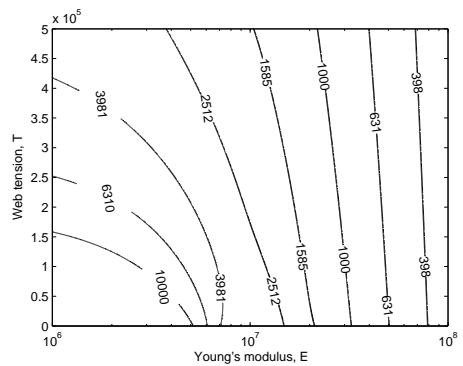

(c)

Fig. 3: Maximum displacement of the web for a range of web stiffnesses (Young's modulus, $E$ ) and web tensions, $T$, for a roll-to-web gap of $g=0.05$. Contour plots are for pickouts of (a) $\Phi=0.0$, (b) $\Phi=0.5$ and (c) $\Phi=1.0$. Contour values $\times 10^{-6}$

gap is shown as a dotted line - as expected this coincides with the minimum web deformation for each configuration. As the roll-to-web gap varies, the pickout at which this occurs changes, and since the roll-to-web gap will vary throughout the entire coating bead it is not sufficient to assume that the pickout for the entire process is that at which the minimum pressure difference occurs.

Figure 3 reveals how the maximum displacement varies with Young's modulus and web tension, from which it can be clearly seen that the maximum web displacement occurs for the lowest web stiffness. From numerical results, when the Young's moduli exceeds $E \approx 10^{7}$ the web tension has a smaller effect on web deformation than Young's modulus due to the relative importance of web stiffness when compared with tension. This is also observed for larger rollto-web gaps (the roll-to-web gap shown here is fairly small and is an extreme case) where web deformation reduces as the roll-to-web gap increases.

The results clearly illustrate that on the length-scale of a single cell, the stiffness and thickness of the web prevents significant deformation, thus providing a basis for more realistic models of the process than have been otherwise been derived.

\section{References}

1. N. Kapur, Chemical Engineering Science 58(13), (2003) 2875-2882

2. H. Benkreira and O. Cohu, Chemical Engineering Science 53(6), (1998) 1223-1231

3. S. R. Rees, PhD thesis, University of Leeds, (1995)

4. L. W. Schwartz, Journal of Engineering Mathematics 42(3-4), (2002) 243-253

5. C. A. Powell, M. D. Savage, and P. H. Gaskell, Chemical Engineering Research and Design 78(A1), (2000) 61-67

6. X. Y. Yin and S. Kumar, Physics of Fluids 17(6), (2005) 063101

7. X. Y. Yin and S. Kumar, Physics of Fluids 18(6), (2006) 063103 